\title{
IRREDUCIBLE ALGEBRAS OF OPERATORS WHICH CONTAIN A MINIMAL IDEMPOTENT
}

\author{
BRUCE A. BARNES ${ }^{1}$
}

\begin{abstract}
We prove that when $A$ is a closed subalgebra of the bounded operators on a reflexive Banach space $X$, which acts irreducibly on $X$ and contains a minimal idempotent, then every bounded operator with finite dimensional range on $X$ is in $A$. We use this result to prove that every continuous irreducible representation of a GCR-algebra on a Hilbert space $\mathcal{H C}$ is similar to a *-representation on $\mathcal{H C}$.
\end{abstract}

1. Notation and terminology. Assume that $X$ is a normed linear space. $B(X)$ denotes the algebra of bounded operators on $X, \mathfrak{C}(X)$ denotes the algebra of compact operators on $X$, and $\mathscr{F}(X)$ denotes the algebra of bounded operators on $X$ which have finite dimensional range. A subalgebra $A$ of $B(X)$ acts irreducibly on $X$ (or is irreducible on $X$ ) if the only closed $A$-invariant subspaces of $X$ are 0 and $X . A$ acts strictly irreducibly on $X$ if the only $A$-invariant subspaces of $X$ are 0 and $X$.

If $A$ is a normed algebra and $X$ is a normed linear space, then a representation of $A$ on $X$ is an algebra homomorphism $\pi$ of $A$ into $B(X)$. A representation $\pi$ of $A$ on $X$ is irreducible (strictly irreducible) if $\pi(A)$ acts irreducibly (strictly irreducibly) on $X$. When $A$ has an involution $*$ and $\mathcal{H}$ is a Hilbert space, a representation $\pi$ of $A$ on $\mathcal{H C}$ is a ${ }^{*}$-representation if $\pi\left(a^{*}\right)=\pi(a)^{*}$ for all $a \in A$.

Let $X$ be a normed linear space. We denote the dual space of $X$ as $X^{*}$. Given $x \in X$ and $f \in X^{*}$, let $(f \mid x)$ be the operator defined by $(f \mid x)(y)=f(y) \cdot x, y \in X$. Every bounded operator on $X$ with 1 dimensional range has the form $(f \mid x)$ for some $x \in X, f \in X^{*}$. When $\mathcal{F}$ is a Hilbert space and $\phi, \psi \in \mathcal{H}$, then $(\phi \mid \psi)$ is the operator defined by $(\phi \mid \psi)(\tau)=(\tau, \phi) \cdot \psi, \tau \in \mathfrak{F}$.

A nonzero idempotent $E$ in a complex normed algebra $A$ is a minimal idempotent of $A$ if $E A E=\{\lambda E \mid \lambda$ complex $\}$. There is a close relationship between minimal idempotents of $A$ and minimal left (or right) ideals of $A$; see [5, pp. 45-46]. All vector spaces in this paper are complex.

Received by the editors July 30, 1970 .

AMS 1970 subject classifications. Primary 46L20, 46L05.

Key words and phrases. Algebras of operators, irreducible algebra, irreducible representation, $B^{*}$-algebra.

${ }^{1}$ A portion of this research was supported by a grant from the Graduate School of the University of Oregon. 
2. Irreducible algebras of operators which contain a minimal idempotent. We assume throughout this section that $X$ is a normed linear space and that $A$ is a subalgebra of $B(X)$ such that $A$ acts irreducibly on $X$. We prove several lemmas and then the main result of this section (Theorem 3).

LEMMA 1. If $E$ is a minimal idempotent of $A$, then there exist $x \in X$, $f \in X^{*}$, with $f(x)=1$, such that $E=(f \mid x)$.

Proof. Choose $x \in X, x \neq 0$, such that $E(x)=x$. Let $Y=\{T(x) \mid T \in A\} . Y$ is an invariant subspace of $X$ for $A$. When $T \in A$ we denote by $T^{\prime}$ the restriction of $T$ to $Y$. Assume that $T_{1}(x)$ and $T_{2}(x)$ are in the range of $E^{\prime}, T_{1}, T_{2} \in A$. Then $T_{i}(x)=E^{\prime} T_{i}(x)$ $=E T_{i} E(x)=\lambda_{i} x$ for some scalars $\lambda_{i}, i=1,2$. Thus $E^{\prime}$ has 1-dimensional range on $Y$. It follows that $E^{\prime}=(g \mid x)$ for some $g \in Y^{*}$. Also since $E^{\prime}$ is a projection, then $g(x)=1$. Let $f$ be the unique extension of $g$ to a continuous functional on $X$ (note that $Y$ is dense in $X$ ). Then $E=(f \mid x)$ since $E-(f \mid x)$ is 0 on $Y$.

Lemma 2. Assume that $A$ is a closed subalgebra of $B(X)$ and that $A$ contains a minimal idempotent $E$. Then $A$ acts strictly irreducibly on $X$.

Proof. By Lemma $1, E=(f \mid x)$ for some $x \in X, f \in X^{*}$, with $f(x)$ 1. $A E=\{(f \mid T(x)) \mid T \in A\}$ is a left ideal of $A$ which is closed in the operator norm. $W=\{T(x) \mid T \in A\}$ is a nonzero invariant subspace of $X$ for $A$, and, therefore, $W$ is dense in $X$. Given $y \in X$ we can choose $\left\{y_{n}\right\} \subset W$ such that $y_{n}=T_{n}(x) \rightarrow y$. Then $\left(f \mid y_{n}\right)=T_{n}(f \mid x)$ $\in A E$ for all $n$ and $\left(f \mid y_{n}\right) \rightarrow(f \mid y)$ in the operator norm. Therefore $(f \mid y) \in A E$. Assume now that $y_{1}, y_{2} \in X$ and $y_{1} \neq 0$. As above $\left(f \mid y_{1}\right)$, $\left(f \mid y_{2}\right) \in A E . \mathscr{L}=A\left(f \mid y_{1}\right)$ is a nonzero left ideal of $A$ in $A E$. By the proof of [5, Lemma (2.18), p. 45], $\mathfrak{L}=A E$. Then there exists $T \in A$ such that $T\left(f \mid y_{1}\right)=\left(f \mid y_{2}\right)$. It follows that $T\left(y_{1}\right)=y_{2}$, and therefore $A$ acts strictly irreducibly on $X$.

Theorem 3. Assume that $X$ is a reflexive Banach space, and that $A$ is a closed subalgebra of $\mathrm{B}(X)$ which acts irreducibly on $X$ and contains a minimal idempotent $E$. Then $\mathfrak{F}(X) \subset A$.

Proof. By Lemma 1 there exists $x \in X, f \in X^{*}$ with $f(x)=1$ such that $E=(f \mid x)$. By Lemma 2, $A$ acts strictly irreducibly on $X$. Therefore $(f \mid y) \in A$ for all $y \in X$. Let $K=\left\{g \in X^{*} \mid(g \mid y) \in A\right.$ for some $y \in X, y \neq 0\}$. We prove that $K$ is a closed subspace of $X^{*}$. For assume that $f_{1}, f_{2} \in K$. Then there exists $x_{1} \neq 0, x_{2} \neq 0$ in $X$ such that $\left(f_{1} \mid x_{1}\right)$ and $\left(f_{2} \mid x_{2}\right)$ are in $A$. Choose $T \in A$ such that $T\left(x_{1}\right)=x_{2}$. Then $\left(f_{1}+f_{2} \mid x_{2}\right)=T\left(f_{1} \mid x_{1}\right)+\left(f_{2} \mid x_{2}\right)$. Thus, $f_{1}+f_{2} \in K$. Now assume that 
$\left(f_{n} \mid x_{n}\right) \in A, x_{n} \neq 0$ for all $n$, and $f_{n} \rightarrow g$ in $X^{*}$. Choose $T_{n} \in A$ such that $T_{n}\left(x_{n}\right)=x$ for all $n$. Then $T_{n}\left(f_{n} \mid x_{n}\right)=\left(f_{n} \mid x\right) \in A$ for all $n$ and $\left(f_{n} \mid x\right)$ $\rightarrow(g \mid x)$ in $\mathbb{B}(X)$. Since $A$ is closed, $(g \mid x) \in A$, and this proves $g \in K$.

Now suppose that $K \neq X^{*}$. Since $K$ is closed and $X$ is reflexive, there exists $y \in X, y \neq 0$, such that $g(y) \neq 0$ for all $g \in K$. Given $T \in B(X)$, let $T^{*}$ be the conjugate (adjoint) operator of $T$ on $X^{*}$. Note that $(f \mid x) T=\left(T^{*}(f) \mid x\right)$. Then $T^{*}(f) \in K$ for all $T \in A$, and it follows that $T^{*}(f)(y)=f(T(y))=0$ for all $T \in A$. But there exists $T \in A$ such that $T(y)=x$, and $f(x)=1$. This contradiction proves that $K=X^{*}$. Therefore, by the definition of $K, A$ contains every bounded operator on $X$ which has 1-dimensional range. This implies that $\mathcal{F}(X) \subset A$, so the proof of the theorem is complete.

We denote the radical of an algebra $B$ by $\operatorname{rad}(B)$. In the next theorem we give a sufficient condition that an irreducible algebra $A$ contain a minimal idempotent.

Theorem 4. Assume that $X$ is a Banach space and that $A$ is a closed subalgebra of $B(X)$ which acts irreducibly on $X$. If $A$ contains an operator $C \in \mathrm{e}(X)$ such that $C$ does not have zero spectrum, then $A$ contains a minimal idempotent.

Proof. $A \cap \mathfrak{e}(X)$ is a closed subalgebra of $\mathfrak{e}(X)$ which contains $C$. Since $C$ does not have zero spectrum, we can produce a nonzero projection $F \in A \cap \mathfrak{e}(X)$ by taking the appropriate contour integral about a nonzero (isolated) point of the spectrum of $C$. F must have finite dimensional range. Then $F A F$ is a finite dimensional subalgebra of $A$. By the Wedderburn theory there exists a projection $E$ in $F A F$ such that the residue class of $E$ in the quotient algebra $F A F / \operatorname{rad}(F A F)$ is a minimal idempotent. $\operatorname{rad}(F A F)$ is nilpotent, so we can choose a positive integer $m$ such that $(F S F)^{m}=0$ whenever $F S F \in \operatorname{rad}(F A F) . \quad$ By $[2, \quad$ Proposition 1, p. 48], $\operatorname{rad}(F A F)$ $=F \operatorname{rad}(A) F$. Then if $T \in \operatorname{rad}(A)$, we have $E T E \in \operatorname{rad}(F A F)$. Therefore $(E T E)^{m}=0$ whenever $T \in \operatorname{rad}(A)$.

Now we show that $\operatorname{rad}(A) \cap A E=0$. For suppose not. Then there exists $T \in \operatorname{rad}(A) \cap A E$ such that $T=T E \neq 0$. Choose $x \in X, x \neq 0$, such that $E(x)=x$ and $T(x) \neq 0$. Set $M=\operatorname{rad}(A) \cap A E$. If $S \in M$, then $S^{m+1}=S(E S E)^{m}=0$. The set $\{S(x) \mid S \in M\}$ is a nonzero invariant subspace of $X$ for $A$. Therefore there exists $\left\{S_{n}\right\} \subset M$ such that $S_{n}(x) \rightarrow x . E M(x)$ is a finite dimensional (and hence closed) subspace of $X$. Since $E S_{n}(x) \rightarrow E(x)=x, x \in E M(x)$. Therefore there exists $S \in M$ such that $E S(x)=x$. But $E S \in M$, so that $(E S)^{m+1}=0$. This is a contradiction since $0 \neq x=(E S)^{m+1}(x)=0$. Therefore $\operatorname{rad}(A) \cap A E=0$. 
Given $T \in A$ there exists a scalar $\lambda$ and $S \in \operatorname{rad}(F A F)$ such that $E T E=\lambda E+S$ (recall that $E+\operatorname{rad}(F A F)$ is a minimal idempotent in $F A F / \operatorname{rad}(F A F))$. Then $E T E-\lambda E=S \in \operatorname{rad}(A) \cap A E=0$. Therefore $E$ is a minimal idempotent of $A$.

Corollary 5. Assume that $A$ satisfies the hypotheses of Theorem 4. Then $A$ acts strictly irreducibly on $X$.

Corollary 6. Assume that $A$ satisfies the hypotheses of Theorem 4 and that $X$ is a reflexive Banach space. Then $\mathcal{F}(X) \subset A$.

3. Representations similar to *-representations. In this section we give a sufficient condition that a continuous irreducible representation of a $B^{*}$-algebra on a Hilbert space $\mathfrak{H C}$ be similar to a *representation on $\mathfrak{H C}$. R. V. Kadison gives very general necessary and sufficient conditions in [3]. Kadison also discusses the important connections this subject has with the representation theory of topological groups.

Throughout this section we assume that $A$ is a $B^{*}$-algebra. S. Cleveland has shown, [1, Lemma 5.3, p. 1104], that when $\pi$ is a continuous algebra isomorphism of $A$ into a Banach algebra $B$, then $\pi(A)$ is closed in $B$. This is easily extended to the case where $\pi$ has a nonzero kernel $I$. For since $\pi$ is continuous, $I$ is a closed ideal of $A$, and then $A / I$ is again a $B^{*}$-algebra. Define $\tilde{\pi}$ on $A / I$ by $\tilde{\pi}(a+I)$ $=\pi(a), a \in A . \tilde{\pi}$ is a continuous algebra isomorphism of $A / I$ into $B$. Therefore, the range of $\tilde{\pi}$ is closed in $B$ by Cleveland's result. But $\pi(A)=\tilde{\pi}(A)$, so that $\pi(A)$ is closed in $B$.

Throughout this section $\mathcal{H C}$ denotes a Hilbert space. Assume that $\pi$ is a continuous irreducible representation of the $B^{*}$-algebra $A$ into $B(\mathcal{F C})$, and that $A / \operatorname{ker}(\pi)$ contains a minimal idempotent. Then $\pi(A)$ is an irreducible closed subalgebra of $B(\mathcal{H C})$ which contains a minimal idempotent. An application of Theorem 3 proves the following result.

Lemma 7. A ssume that $\pi$ is a continuous irreducible representation of $A$ on $\mathcal{F}$ such that $A / \operatorname{ker}(\pi)$ contains a minimal idempotent. Then $\pi$ is strictly irreducible on $\mathfrak{H C}$ and $\mathrm{e}(\mathcal{H C}) \subset \pi(A)$.

Every minimal left ideal of $A$ has the form $A h$ where $h$ is a selfadjoint minimal idempotent by [5, Lemma (4.10.1), p. 261]. Assume that $h$ is a selfadjoint minimal idempotent of $A$. Then $h A h$ is just the set of all scalar multiples of $h$. We define an inner product $\langle\cdot, \cdot\rangle$ on $A h$ by the rule $\langle x h, y h\rangle h=h y^{*} x h$; see [5, Theorem (4.10.3), p. $261]$. We call $\langle\cdot, \cdot\rangle$ the canonical inner product on $A h$. Now we prove the main result of this section. 
TheOREM 8. Assume that $A$ is a $B^{*}$-algebra and that $\pi$ is a continuous irreducible representation of $A$ on 3 . Assume that $A / \operatorname{ker}(\pi)$ contains a minimal left ideal. Then there exists a strictly irreducible *-representation $\rho$ of $A$ on $\mathfrak{H C}$ and a positive invertible operator $V \in B(\mathcal{H C})$ such that $\pi(a)=V^{-1} \rho(a) V$ for all $a \in A$.

Proof. We may assume without loss of generality that $\pi$ is an isomorphism. For in the general case we can define $\tilde{\pi}$ on $A / \operatorname{ker}(\pi)$ as in the discussion preceding Lemma 7 , and apply our arguments to $\tilde{\pi}$. Therefore we assume that $\pi$ is an isomorphism and that $A$ has a minimal left ideal $A h$, where $h$ is a selfadjoint minimal idempotent of $A$. Let $\langle\cdot, \cdot\rangle$ denote the canonical inner product on $A h$ and let $(\cdot, \cdot)$ denote the inner product on $\mathcal{H C} . \pi(h)$ is a minimal idempotent of $\pi(A)$. It follows that there exists $\phi, \psi \in \mathcal{F}$ such that $(\psi, \phi)=1$ and $\pi(h)=(\phi \mid \psi)$. By Lemma $7, \pi$ is strictly irreducible on $\mathcal{H}$. Since $\pi(A h) \psi$ is a nonzero invariant subspace of $\mathcal{H}$ for $\pi(A)$, then $\mathcal{H}$ $=\pi(A h) \psi$. If, for some $x \in A, \pi(x h) \psi=0$, then $\pi(x)(\phi \mid \psi) \psi=0$, and therefore $\pi(x) \psi=0$. Then $\pi(x)(\phi \mid \psi)=0$, so that $\pi(x h)=0$, and finally we have $x h=0$. Therefore $x h \rightarrow \pi(x h) \psi$ is a 1-1 map of $A h$ on to F. We use this map to transfer the canonical inner product on $A h$ to a positive definite form on $\mathcal{H}$. Define $[\pi(x h) \psi, \pi(y h) \psi]=\langle x h, y h\rangle$ for all $x h, y h \in A h$. It follows from this definition that $[\cdot, \cdot]$ is a bounded positive definite form on $\mathcal{H}$. By Riesz's theorem there exists a positive operator $U \in B(\mathcal{F C})$ such that $\left[\phi_{1}, \phi_{2}\right]=\left(U \phi_{1}, \phi_{2}\right)$ for all $\phi_{1}, \phi_{2} \in \mathcal{H}$. Assume that $\tau=\pi(x h) \psi \in \mathcal{H}$. Then

$$
\begin{aligned}
(\tau, \tau) & =(\pi(x h) \psi, \pi(x h) \psi) \leqq\|\pi(x h)\|^{2}\|\psi\|^{2} \leqq\|\pi\|^{2}\|\psi\|^{2}\|x h\|^{2} \\
& =\|\pi\|^{2}\|\psi\|^{2}\left\|h x^{*} x h\right\|=\|\pi\|^{2}\|\psi\|\left\|^{2}\right\| h \|\langle x h, x h\rangle .
\end{aligned}
$$

Set $M=\|\pi\|^{2}\|\psi\|^{2}\|h\|$. We have

$$
(\tau, \tau) \leqq M\langle x h, x h\rangle=M[\pi(x h) \psi, \pi(x h) \psi]=M[\tau, \tau]=M(U \tau, \tau) .
$$

Therefore $U^{-1} \in \Re(\mathcal{H C})$.

Let $V=\sqrt{ } U$. Define $\rho(a)=V \pi(a) V^{-1}, a \in A$. Given $\psi_{1}, \psi_{2} \in \mathfrak{H}$, there exists $\phi_{1}, \phi_{2} \in \mathcal{H C}$ and $x_{1}, x_{2} \in A$ such that $\psi_{i}=V \phi_{i}$ and $\phi_{i}=\pi\left(x_{i} h\right) \psi$ for $i=1,2$. Then

$$
\begin{aligned}
\left(\rho(a) \psi_{1}, \psi_{2}\right) & =\left(V \pi(a) V^{-1} V \phi_{1}, V \phi_{2}\right)=\left[\pi(a) \phi_{1}, \phi_{2}\right] \\
& =\left[\pi(a) \pi\left(x_{1} h\right) \psi, \pi\left(x_{2} h\right) \psi\right]=\left\langle a x_{1} h, x_{2} h\right\rangle \\
& =\left\langle x_{1} h, a^{*} x_{2} h\right\rangle=\left[\pi\left(x_{1} h\right) \psi, \pi\left(a^{*}\right) \pi\left(x_{2} h\right) \psi\right] \\
& =\left(V \phi_{1}, V \pi\left(a^{*}\right) V^{-1} V \phi_{2}\right)=\left(\psi_{1}, \rho\left(a^{*}\right) \psi_{2}\right) .
\end{aligned}
$$


Therefore $\rho$ is a $^{*}$-representation of $A$ on $\mathcal{H}$. This completes the proof of the theorem.

Now assume that $A$ is a GCR-algebra as defined by I. Kaplansky; see [4]. Assume that $\pi$ is a continuous irreducible representation of $A$ on a Hilbert space $\mathcal{H}$. Then $A / \operatorname{ker}(\pi)$ has no ideal divisors of zero by $[4$, Lemma 2.5, p. 223]. Therefore $A / \operatorname{ker}(\pi)$ contains a minimal left ideal by [4, Lemma 7.4, p. 247]. These remarks together with Theorem 8 prove the following corollary.

Corollary 9. When $A$ is a GCR-algebra, then every continuous irreducible representation of $A$ on a Hilbert space $\mathcal{H C}$ is similar to a strictly irreducible *-representation of $A$ on $\mathfrak{H C}$.

\section{REFERENCES}

1. S. B. Cleveland, Homomorphisms of non-commutative *-algebras, Pacific J. Math. 13 (1963), 1097-1109. MR 28 \#1500.

2. N. Jacobson, Structure of rings, Amer. Math. Soc. Colloq. Publ., vol. 37, Amer. Math. Soc., Providence, R. I., 1956. MR 18, 373.

3. R. V. Kadison, On the orthogonalization of operator representations, Amer. J. Math. 77 (1955), 600-620. MR 17, 285.

4. I. Kaplansky, The structure of certain operator algebras, Trans. Amer. Math. Soc. 70 (1951), 219-255. MR 13, 48.

5. C. E. Rickart, Banach algebras, University Series in Higher Math., Van Nostrand, Princeton, N. J., 1960. MR 22 \#5903.

University of Oregon, Eugene, Oregon 97403 